\title{
Sistema Computacional para Aplicação do Modelo Parabólico de Praias de Enseada*
}

\author{
Ariel Vargas ${ }^{1}$; André L. Alice Raabe ${ }^{2}$ e Antonio H. da Fontoura Klein ${ }^{3}$ \\ Universidade do Vale do Itajaí Centro de Ciências Tecnológicas da Terra e do Mar CTTMar \\ Rua Uruguai, 458 - Bairro Centro, Itajaí - SC - Brasil - Cep: 88.302-202 Fone/Fax: (47) 341-7544

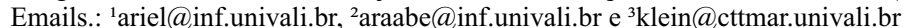 \\ * Projeto Financiado pelo Artigo 170- Governo do Estado de Santa Catarina e UNIVALI-ProPPEX e CTTMAR \\ + Instituição integrante do Programa de Geologia e Geofísica Marinha PGGM \\ Recebido 28 de dezembro 2001; revisado 12 de junho 2002; aceito 19 de julho 2002
}

\begin{abstract}
RESUMO
As praias de enseada constituem a morfologia litorânea predominante no Estado de Santa Catarina. Através de modelos empíricos é possível definir e analisar a linha de costa, em planta, além de prever possíveis alterações em sua localização e formato. O Modelo Parabólico é um modelo empírico que permite determinar a situação de equilíbrio morfodinâmico das praias de enseada. Devido a realização dos cálculos do modelo e posterior análise dos resultados revelarem-se tarefas trabalhosas e demoradas, construiu-se um sistema computacional para auxiliar na realização dessas tarefas, além de permitir a visualização dos resultados através de simulação gráfica. O sistema, denominado MEPPE, foi construído em linguagem Object Pascal, com recursos de programação visual, para ser utilizado em plataforma Windows. A partir de um conjunto de informações fornecidas pelo usuário, o programa desenha a linha de costa teórica de uma praia conforme o Modelo Parabólico. Com isso, é possível estabelecer uma análise da situação de equilíbrio ou desequilíbrio da praia analisada, comparando-se a linha de costa existente com a linha de costa teórica encontrada. A utilização do MEPPE possibilita aproximar os conceitos teóricos, trabalhados em sala de aula, de sua utilização prática, permitindo melhor compreensão do tema, despertando maior interesse nos alunos. O programa proporciona um ambiente de experimentação onde a manipulação direta das informações e a visualização imediata dos resultados contribui para um melhor aprendizado do assunto.
\end{abstract}

Palavras chave: Processos costeiros, praias de enseada, modelo parabólico.

\begin{abstract}
The headland bay beaches constitute the predominant coastal morphology in Santa Catarina State. The empiric Parabolic Model allows the definition and analyzis of the planform shape of these beaches. It also predicts possible changes in its location and format. As the accomplishment of Parabolic model calculations and subsequent analysis are slow and workfull, software was developed to aid the task allowing the visualization of results through graphic simulation. The software named MEPPE was developed in Object Pascal language using visual programming resources based on MS-Windows platform. It uses a set of information supplied by the user for drawing the theoretical coastline of a beach according to the Parabolic Model. So, it's possible to establish an analysis of the situation of balance or imbalance of the analyzed beach, providing a comparison between the existent line and the theoretical coastline. MEPPE makes possible to approach theoretical concepts, studied in classroom, with a practical manner giving a better comprehension of the theme and increasing the interest of students. The software contributes to a better learning of the subject providing the direct manipulation of parameters and the immediate visualization of results.
\end{abstract}

Keywords: Coastal processes, headland bay beaches, parabolic model

\section{Introdução}

Praias de enseada são muito comuns no Brasil, principalmente no litoral catarinense. Por definição, são praias limitadas por promontórios rocho-sos ou outros obstáculos físicos, geralmente forman-do um arco com curvatura acentuada e cujo contorno tende a assumir a forma de um meio coração ou de uma lua crescente (Short e Masselink, 1999).
As praias de enseada, na maioria das vezes, desenvolvem formas assimétricas, tendo como característica uma zona de sombra localizada próxima ao promontório rochoso, protegida da energia de on-das e fortemente curvada (Figura 1). A parte central é levemente curvada, e a outra extremidade é relativamente retilínea, sendo normalmente paralela à direção dominante dos trens de onda na região. 
Segundo Silvester e Hsu (1993), as praias de enseada podem apresentar-se em equilíbrio estático ou em equilíbrio dinâmico. Em uma situação estável, ou equilíbrio estático, os trens de onda dominantes atingem toda a extensão da praia em ângulo de $90^{\circ}$, as cristas de onda quebram simultaneamente ao lon-go da praia e o transporte longitudinal de sedimento, bem como os processos de erosão e deposição, são anulados. No entanto, quando há suprimento sedimentar para o local e o transporte longitudinal é ativo, a praia se encontra em um estado instável, de equilíbrio dinâmico. Neste tipo de praia, as forças de deriva litorânea e o suprimento sedimentar são fato-res determinantes na manutenção da faixa de praia na sua posição atual.

As alterações morfológicas são geralmente ocasionadas por fatores que modificam a trajetória natural das ondas que chegam à praia, entre os quais, a existência de barreiras físicas, tais como ilhas ou promontórios rochosos e, principalmente, cons-truções, como molhes e plataformas (Short e Masselink, 1999).

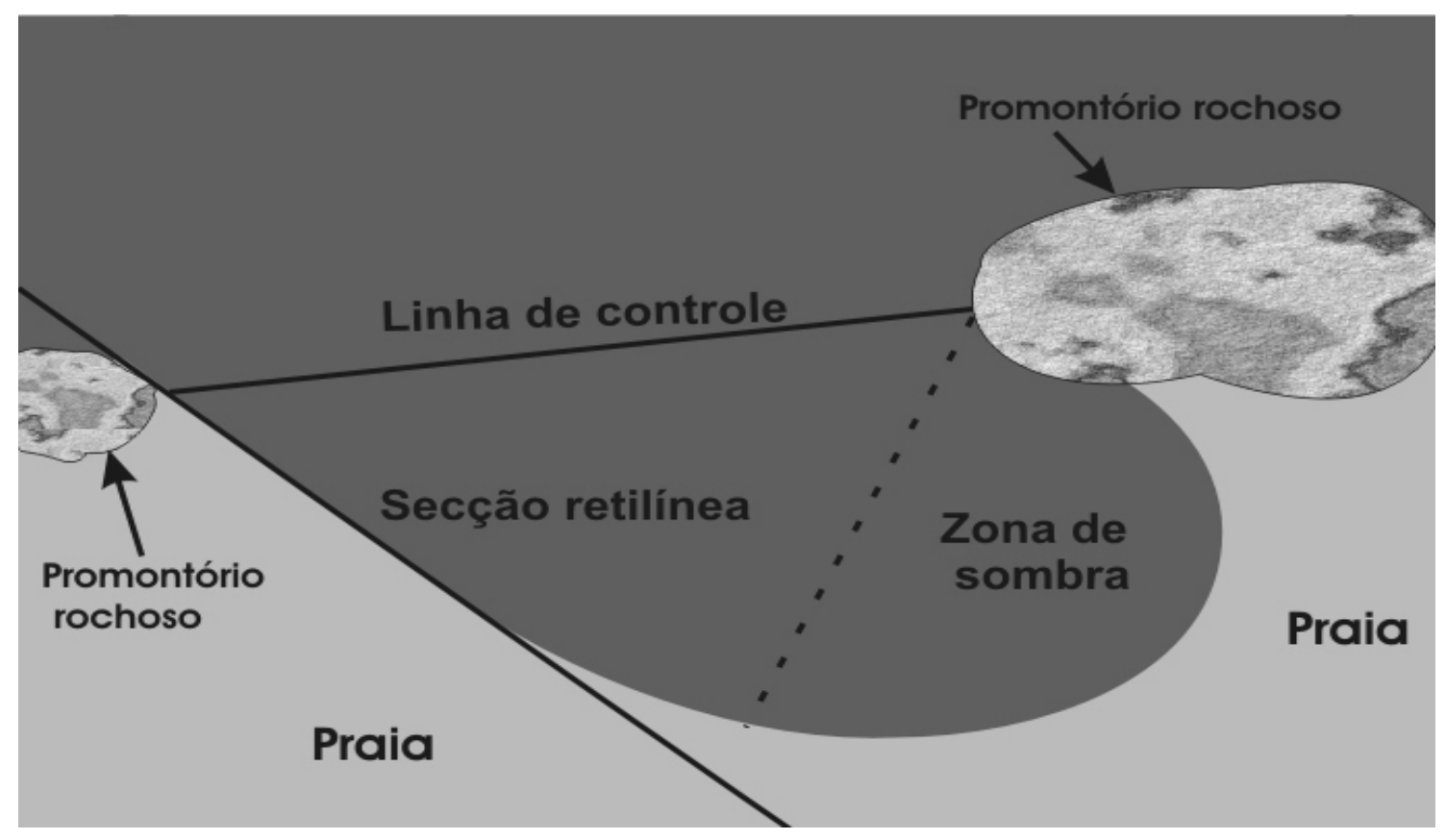

Figura 1. Representação dos principais componentes de uma praia de enseada.

Alguns modelos empíricos são muito eficien-tes na análise e estudo do comportamento das praias de enseada, entre eles o Logaritmo Espiral (Silves-ter, 1970), o Modelo Parabólico (Silvester e Hsu, 1993), e o Modelo Hiperbólico (Moreno e Kraus, 1999). D e acordo com Klein et al. (em publicação), o modelo parabólico apresenta-se mais robusto que os demais por não representar apenas uma solução matemática que define a forma da enseada apenas a partir de dados geométricos, mas leva em consi-deração parâmetros relacionados com a direção da onda de maior energia (obliqüidade).

Analisando-se a aplicação do modelo parabó-lico percebeu-se que esta se revelava trabalhosa e demorada. Constatou-se que a aplicação e análise do modelo poderiam ser agilizadas e facilitadas com o auxílio do processamento computacional, o que jus-tifica a implementação de um sistema computacional que simulasse a aplicação do Modelo de Equilíbrio em Planta de Praias de Enseada (MEPPE) de forma gráfica, facilitando o aprendizado do tema.

\section{O Modelo Parabólico}

O Modelo Parabólico baseia-se nas relações entre as características geométricas da praia em planta e o ângulo de incidência das ondas na praia (Hsu e Evans, 1989; Silvester e Hsu, 1993), representada através da equação 1 :

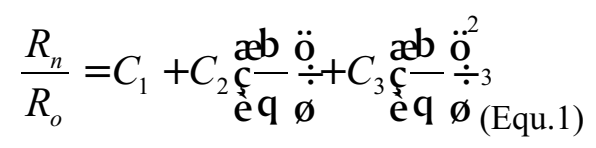

Para a aplicação do modelo extraem-se, através de fotografias aéreas da praia, imagens de satélites ou mapas, os parâmetros descritos na figura 2:

- $\mathrm{R}_{\mathrm{o}}$ ou linha de controle: linha que une o ponto de difração de ondas até o final da parte retilínea da praia;

Linha de direção predominante de ondas: indica a direção predominante de ondas; 
- $\mathrm{R}_{\mathrm{n}}$ raios traçados a partir do promontório e unidos ao longo da praia;

- Ângulo Beta (): ângulo formado entre a linha de direção de ondas predominantes e a linha $\mathrm{R}_{\mathrm{o}}$;

- Ângulos Teta (): ângulos formados entre a linha de direção predominante de ondas e os demais raios $\mathrm{R}_{\mathrm{a}}$

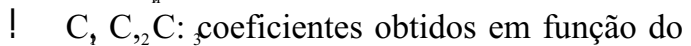
ângulo beta e definidos através de testes e experimentos tabelados (Hsu e Evans, 1989; Silvester e Hsu, 1993).

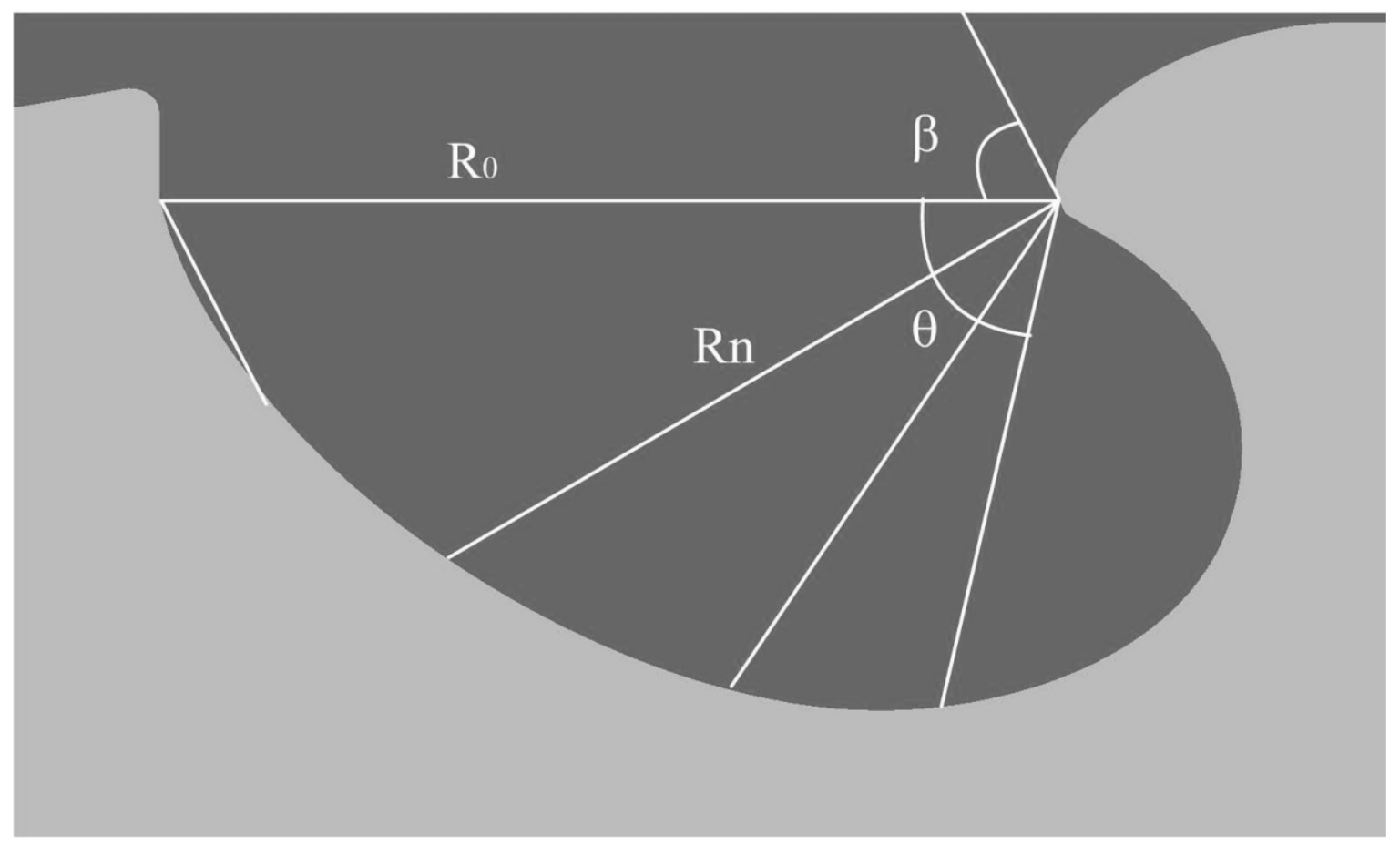

Figura 2. Representação do Modelo Parabólico

\subsection{Análise daAplicação do Modelo Parabólico}

Para a aplicação tradicional do Modelo Parabólico utiliza-se um mapa e cartas topográficas, uma fotografia aérea vertical ou uma imagem de satélite de uma praia, para traçar as retas e obter os ângulos. A aplicação do modelo é adimensional, não importando a escala dos mapas ou cartas topográficas. De posse deste material procede-se da seguinte maneira:

Definição da linha de controle: traça-se uma reta do ponto onde ocorre a difração de ondas (ponto de difração), ou seja, onde existe o obstáculo físico que altera a trajetória inicial das ondas que chegam à praia, até o final da parte retilínea da praia (ponto de retilinidade), ou seja, até o ponto em que o modelo exerce influência na morfologia da linha de costa, efetuando-se a medida do comprimento da reta. Como resultado tem-se o $\mathrm{R}$ 。

Direção predominante de ondas e o ângulo beta: traça-se uma reta sobre a parte mais retilínea da praia e une-se esta reta ao $R_{\diamond}$ sendo o ângulo formado entre estas denominado de ângulo beta, medido em graus.
Os raios $\mathbf{R}_{\mathbf{i}}$ de posse de um transferidor de graus deve-se traçar linhas, de 10 em 10 graus, até um máximo de 150 graus, partindo do ponto de difração de ondas até a linha de costa. Assim, são obtidos os raios $\mathrm{R}_{\mathrm{n}}$ para cada ângulo teta formado.

Determinação do comprimentos dos raios $\mathbf{R}_{\mathrm{n}}$ : o comprimento de cada raio $\mathrm{Rn}$ é encontrado aplicando-se a equação 1, com valores diferentes de C1, C2, e C3 obtidos, em relação ao ângulo (Tabela 1).

A linha teórica de costa: após serem calculados todos os valores dos $\mathrm{R}_{\mathrm{p}}$ para cada ângulo teta, deve-se unir as extremidades de cada raio obtendo-se a Linha de Costa Teórica da praia, em planta.

Com isso, o usuário pode identificar, tendo conhecimentos básicos sobre o assunto, se a praia analisada encontra-se numa situação de equilíbrio estático, onde não ocorrerão modificações morfológicas na linha de costa, ou se a mesma encontrase em equilíbrio dinâmico, onde a linha de costa tende a assumir uma forma diferente da atual.

Baseando-se na análise da tarefa descrita an-teriormente identificou-se a possibilidade de torná-la mais ágil e menos trabalhosa, através do desenvolvi-mento de um sistema computacional. 
Buscou-se de-finir as funcionalidades de um programa que ofe-recesse ao usuário a possibilidade de aplicar o Mo-delo Parabólico com maior facilidade e menor tem-po. Além disso, que fosse possível ao usuário testar diversas condições de praia, realizando comparações entre a situação existente e a situação teórica, segundo o modelo.

Tabela 1: Exemplos da razão Rn/Ro para diferentes valores de B e O (Segundo SilVester e Hsu, 1993)

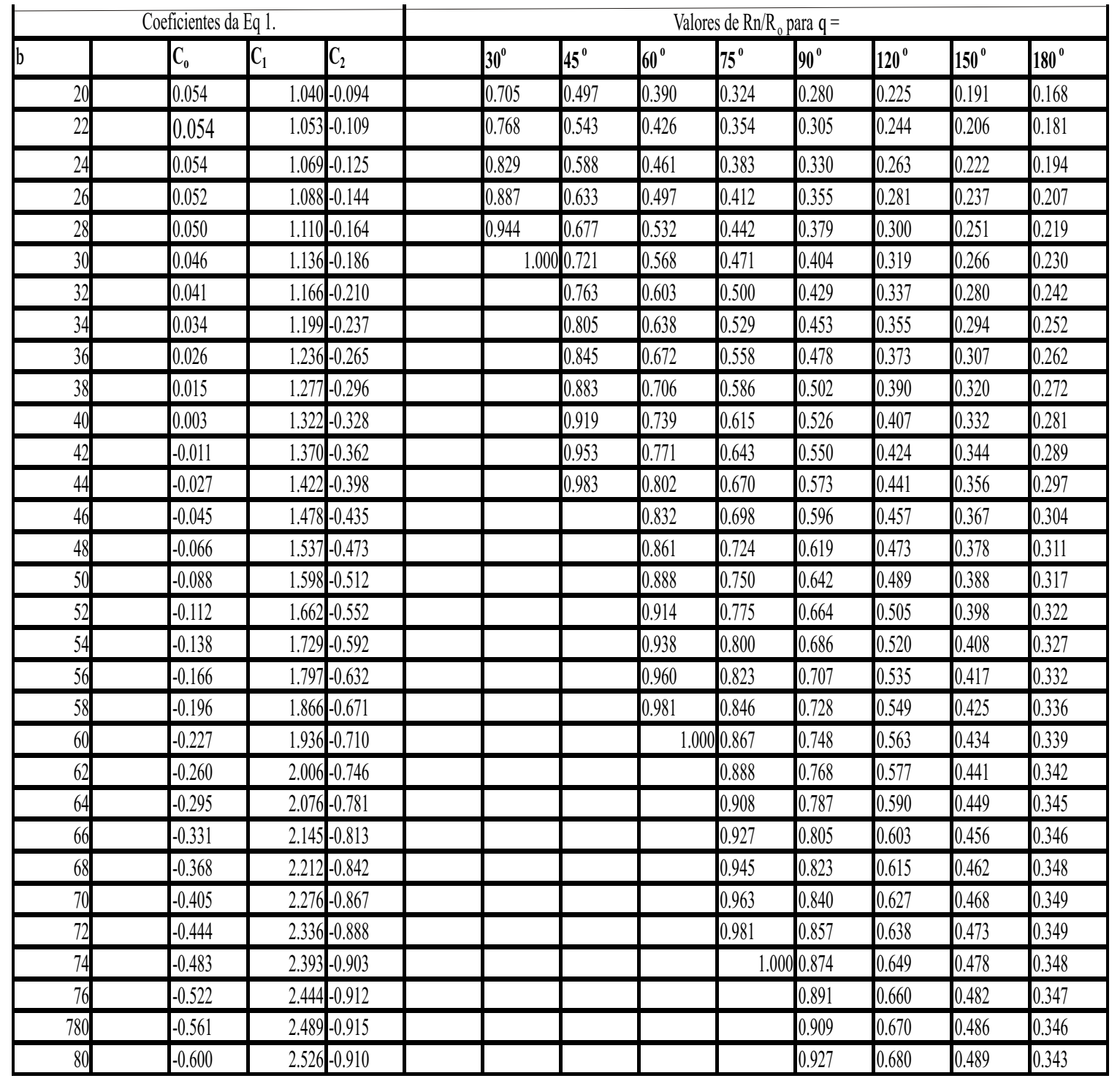

\section{Desenvolvimento}

Para o desenvolvimento do MEPPE foi necessário um trabalho de pesquisa interdisciplinar. Foram realizadas pesquisas referentes aos aspectos teóricos do Modelo Parabólico. Destacaram-se os dialogos com especialistas na área de Processos Cos-teiros, aspecto determinante para a compreensão e domínio do tema, além da indispensável análise dos requisitos para a resolução do problema.

\subsection{Sistema Computacional Educacional}

Para o desenvolvimento de um sistema computacional educacional são necessárias certas estra- tégias que fazem com que o conhecimento não seja simplesmente passado ao usuário, mas sim, que permitam que este se torne construtor do seu próprio conhecimento (Valente, 1989)

.As diretrizes educacionais que nortearam o desenvolvimento do MEPPE foram:

- Buscar a aprendizagem a partir da ação consciente do usuário junto à interface do aplicativo;

Definir o ambiente de forma aberta, ou seja, onde o usuário possui um conjunto indeterminado de possibilidades de exploração, podendo assim refletir sobre as conseqüências de cada ação realizada;

Despertar a curiosidade e 
incentivar um a-profundamento nas questões teóricas trabalhadas na prática.

\subsubsection{Modalidade do MEPPE}

Verificou-se que a modalidade de programa educacional que melhor se adequou às necessidades da tarefa é a de exercício e prática. Segundo Giraffa (2000), os programas de exercício e prática são utilizados como complemento à aula ministrada, on-de o usuário pratica e testa seus conhecimentos

.Na análise da situação de equilíbrio de uma determinada praia faz-se necessário o teste de diver-sas situações para a praia analisada, sendo assim necessário a utilização do sistema como ferramenta de exercício do tema. É neste aspecto que o MEPPE revela-se um programa de exercício e prática.

De acordo com Giraffa (2000), as simulações podem ser caracterizadas pelo fato de serem a exe-cução dinâmica de um modelo previamente definido, recriando uma situação ou ambiente. O sistema, por sua vez, simula a aplicação do modelo parabólico, onde se aplicam suas características de simulação.

\subsection{Implementação Computacional}

Tendo como objetivo permitir ao programa simular graficamente a linha de costa da praia, resultando da aplicação do modelo parabólico, utilizou-se recursos para a manipulação de imagens e a disponibilização de gráficos. Aseguir é descrita a aplicação desses recursos na implementação computacional do modelo.

\subsubsection{Busca da linha de controle $R_{0}$}

O primeiro passo dado na resolução do pro-blema foi a busca do comprimento da linha de con-trole (R). Para tal utilizou-se a equação 2 referente à distância entre dois pontos no plano (Boulos e Camargo, 1987), conforme figura 3.

$$
d^{2}=\left(x_{1}-x_{o}\right)^{2}+\left(y_{1}-y_{o}\right)^{2}
$$

onde:

d - comprimento (em unidades de tela) da linha de controle $\mathrm{R}_{\mathrm{o}}$

$\mathbf{x}_{\mathbf{o}}$ coordenada $\mathrm{x}$ do ponto onde se inicia a reta $R_{\diamond}$ ou seja, o ponto de difração.

$\mathbf{y}_{\mathbf{0}}$ coordenada y do ponto onde se inicia a reta $R_{0}$ reta $R_{0}$

$\mathbf{x}_{1}$ coordenada $\mathrm{x}$ do ponto onde termina a reta $\mathrm{R}_{0}$

$\mathbf{y}_{1}$ coordenada y do ponto onde termina a

Tendo-se o valor de $\mathrm{R}_{0}$, buscou-se encontrar o comprimento da linha de direção predominante de ondas, utilizando a equação (2), para formar com $\mathrm{R}_{\mathrm{o}} \mathrm{o}$ ângulo (beta). Após a obtenção destes valores, foi necessário traçar uma linha virtual ligada ao $\mathrm{R}_{\mathrm{o}} \mathrm{e}$ à linha de direção predominante de ondas a fim de formar um triângulo. Para a obtenção do compri-mento desta linha utilizou-se o mesmo procedimento citado anteriormente.

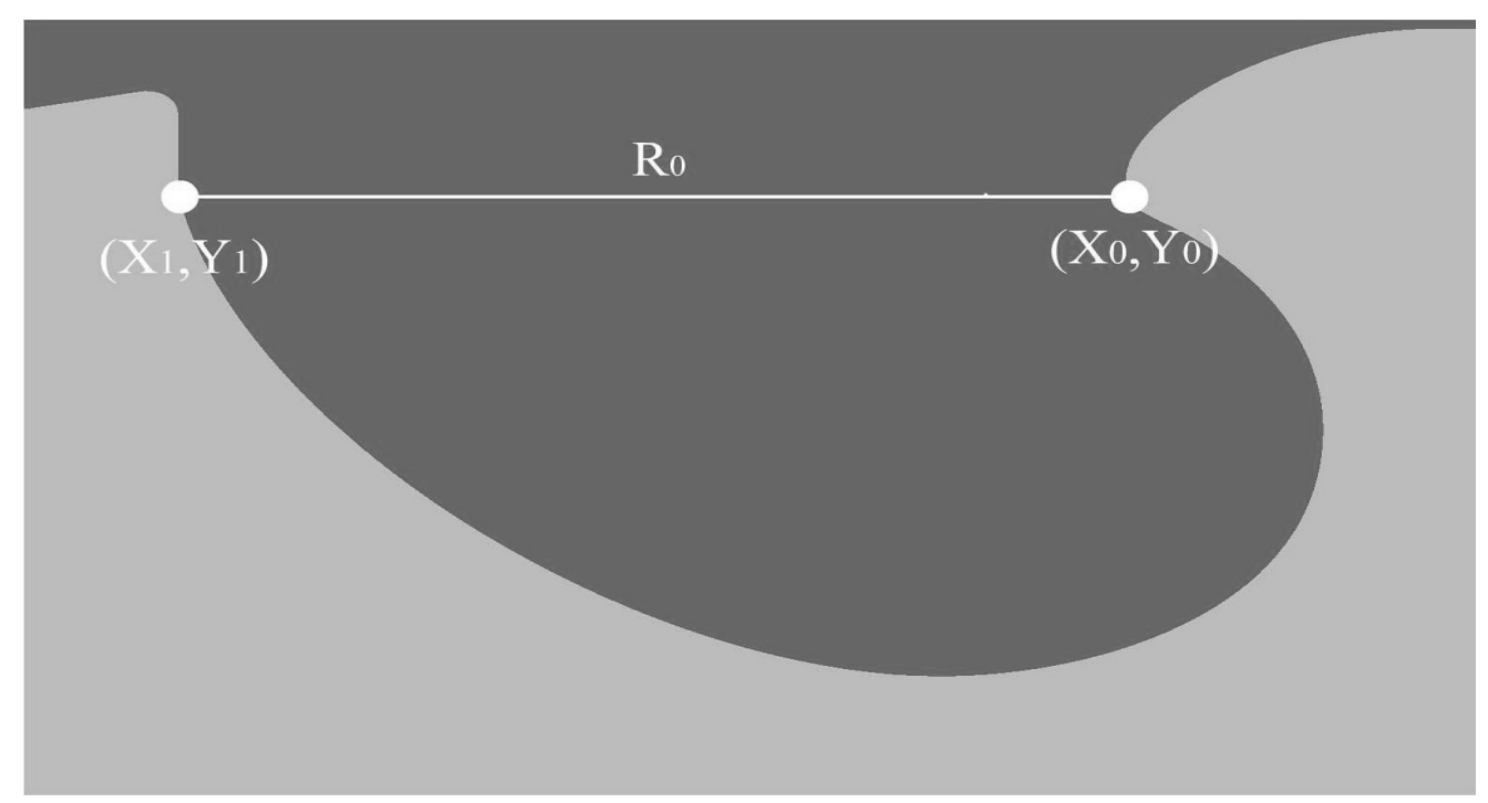

Figura 3. Comprimento do Ro. 


\subsubsection{Cálculo do ângulo}

De posse dos valores dos três lados do triângulo, $\mathrm{R}_{\diamond}$ linha de direção predominante de ondas e a linha virtual, utilizou-se a lei dos cossenos (Boulos e Camargo, 1987) para obter o valor do cosseno de , conforme a equação 3 e a figura 4

$$
|a|^{2}=|b|^{2}+|x|^{2}-2|b||x| \cos \mathbf{b}
$$

Invertendo-se a equação, temos:

$$
\cos \mathbf{b}=\frac{|a|^{2}-|b|^{2}-|x|^{2}}{2|b \| x|}
$$

a comprimento da linha virtual

b comprimento da linha de direção predominante de ondas

\section{x Comprimento do $\mathrm{R}$}

Para encontrar especificamente o valor do ângulo, utilizou-se a função do "Object Pascal”, "arcCos”, (Cantù, 2000), que retorna o arco-cosseno do ângulo, isto é, o seu valor em radianos. Assim, com o valor em radianos foi necessário realizar a conversão para graus, utilizando-se a equação 5 (Boulos e Camargo, 1987):

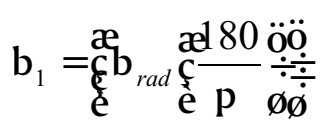

$$
\text { onde: }
$$

${ }_{1}$ - valor do ângulo em graus

rad valor do ângulo em radianos

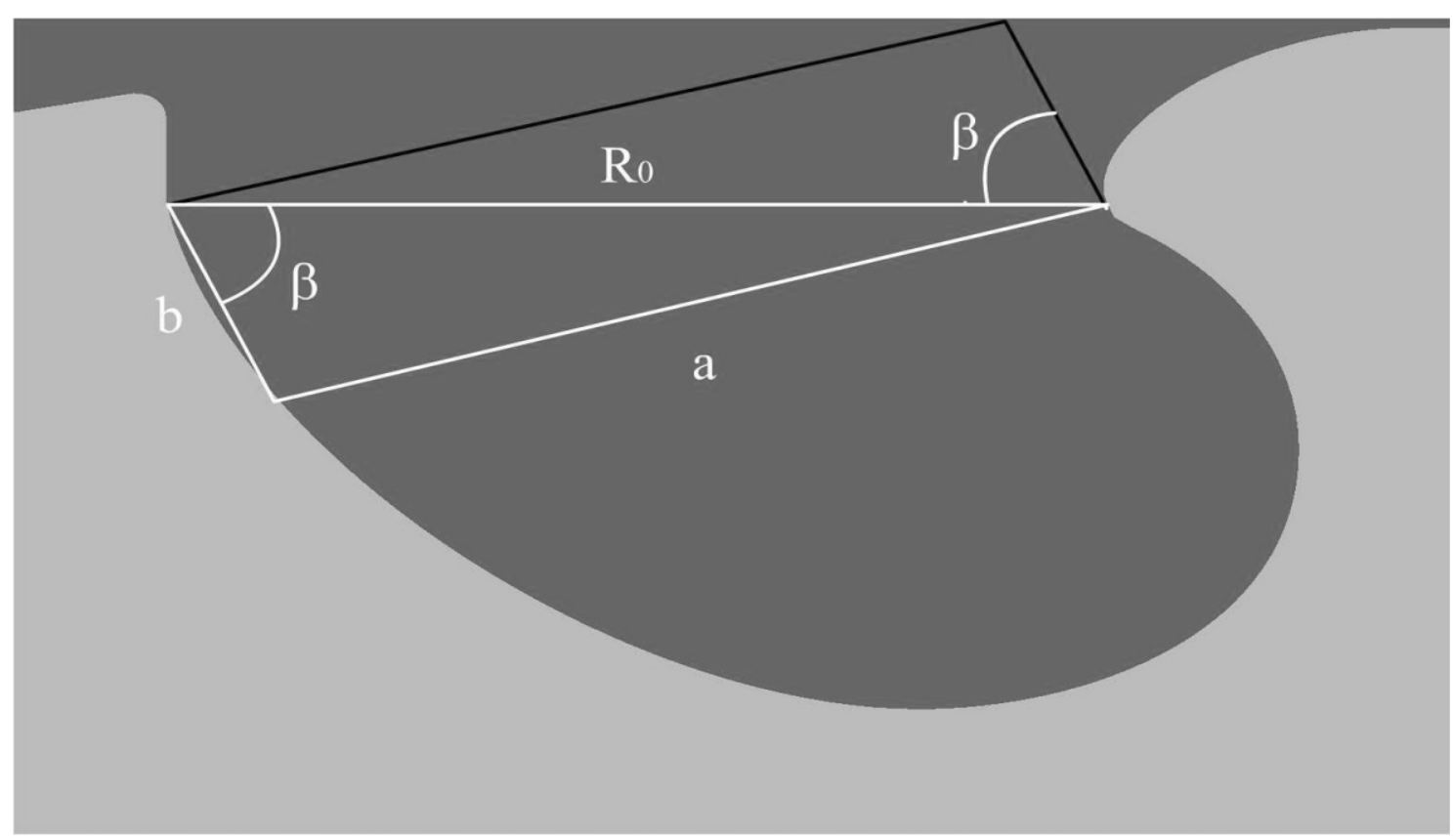

Figura 4. Busca do valor do ângulo beta ().

\subsubsection{Aplicação do Modelo Parabólico}

Possuindo-se os valores de $\mathrm{R}_{\mathrm{o}} \mathrm{e}$, esses valores foram substituídos na equação do modelo parabólico (equação 1) para encontrar os valores dos raios $\mathrm{R}_{\mathrm{h}}$ para cada ângulo .

A variação do ângulo foi implementada utilizando-se uma estrutura de repetição, onde a cada iteração do algoritmo é calculado o valor de um raio $R_{n}$, para seu respectivo ângulo , $e$ armazenado em um vetor. A cada iteração utilizou- se o valor dos coeficientes $\mathrm{C} 1, \mathrm{C} 2, \mathrm{e}_{3}$, armazenados em uma ma-triz bidimensional. Os coeficientes C,C,C sãa definidos pelo modelo e dependem do ângulo beta () em questão.

$\mathrm{O}$ algoritmo que reproduz a aplicação da equação 1 para encontrar os valores dos raios $R_{a}$ apresenta-se descrito no Quadro 1.

\subsubsection{Visualização da Linha Teórica da Costa}

Com a obtenção dos valores para todos os raios $\mathrm{Rn}$, torna-se necessário encontrar sua localização exata na tela, ou seja, saber onde eles 
deve-riam aparecer.

A tela do computador trabalha de maneira similar a um plano cartesiano. Seu ponto $(0,0)$ encontra-se no canto superior esquerdo da tela. A coordenada X (comprimento) aumenta de valor da esquerda para a direita, e a coordenada Y (altura) aumenta de valor de cima para baixo.

Desta maneira, para se encontrar as coordenadas $(\mathrm{X}, \mathrm{Y})$ para os pontos desenhados na tela, necessita-se fazer uma translação de coordenadas, isto é, transportar o ponto origem do plano $(0,0)$ para o ponto onde se situa o ponto de difração de ondas.

Após isso, visto que todas as linhas $\mathrm{R}_{\mathrm{n}}$ tinham a mesma origem, utilizou-se as coordenadas polares
(Anton, 2000) para se determinar os pontos limite de cada raio $\mathrm{R}_{\mathrm{n}}$

Com a equação, foi possível encontrar a coordenada $\mathrm{X}$, do ponto limite do referido raio $\mathrm{R}_{\mathrm{n}}$ Da mesma maneira, utilizando a fórmula , encontrou-se a coordenada $\mathrm{Y}$, do ponto limite do referido $R_{A}$ conforme figura 5 .

Para a obtenção dos pontos de coordenadas $(\mathrm{X}, \mathrm{Y})$ levou-se em conta que o ponto origem foi modificado (translação), assim, para cada ponto encontrado, é necessário converter suas coordenadas para adaptá-las ao ponto origem da tela $(0,0)$, fazendo subtrações ou adições dependendo da localização do ponto de difração.

Quadro 1: A lgoritmoquereproduza aplicaçãodaequação 1 paraencontrarosvaloresdos raios $R_{n}$

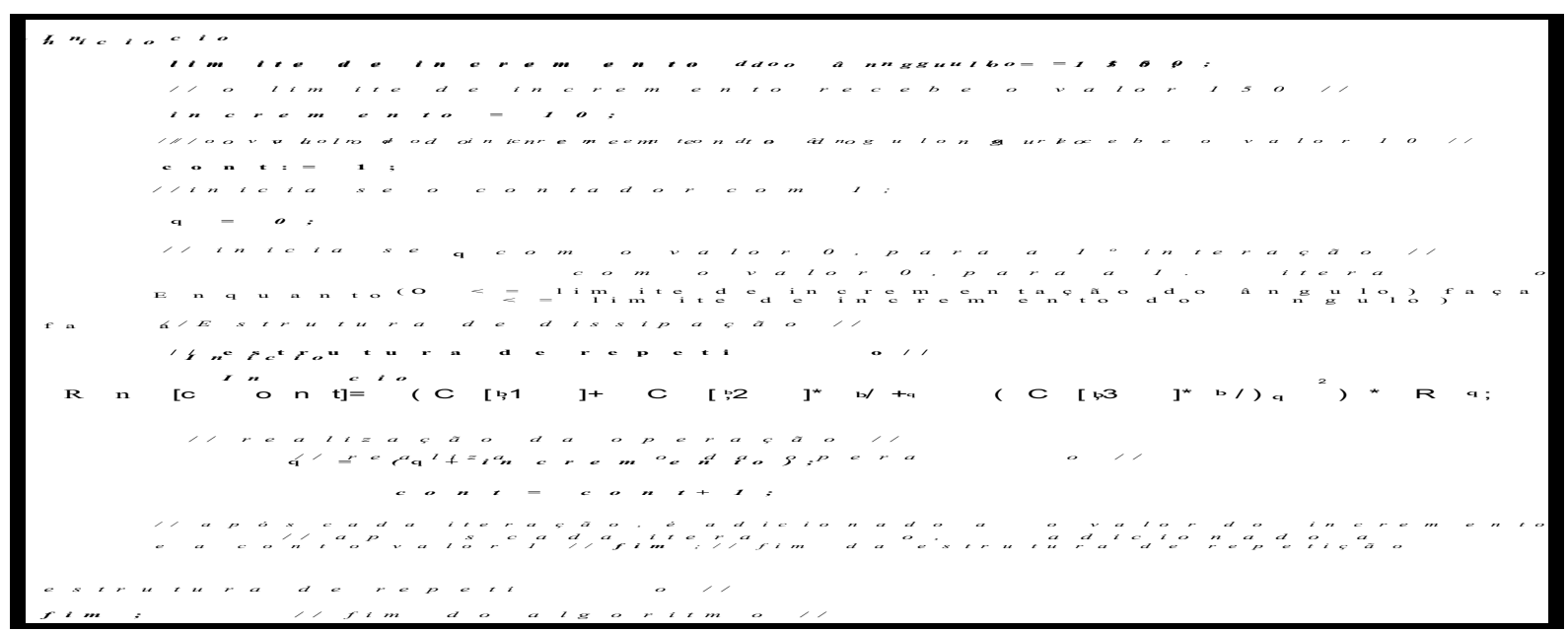

\section{O MEPPE}

O sistema desenvolvido e denominado MEPPE oferece uma interface que permite ao usuário indicar os pontos importantes do Modelo Parabólico, a partir de uma fotografia aérea, uma imagem de satélite, ou mapa de uma praia, em planta (arquivos no formato Raster). O aplicativo possibilita a experimentação de maneira instantânea da representação gráfica da linha de costa teórica, ou seja, onde teoricamente deveria estar a faixa de areia da praia em relação aos pontos selecionados na figura, de acordo com o modelo.

Com a utilização do MEPPE, a implementação do Modelo Parabólico tornou-se uma tarefa mais simples, com um custo de tempo menor, além de apresentar resultados que facilitam a interpretação.

Através dele o usuário pode buscar a aprendi-zagem, a partir de uma ação consciente, pois o resul-tado gerado pelo programa vai depender da seleção dos pontos de definição do modelo, pelo usuário. Este, por sua vez, pode analisar o efeito que deter-minada ação, como a mudança do ponto de difração de ondas, pode causar na morfologia da linha de costa da praia analisada. Assim, pode elaborar suas próprias soluções para os problemas, como o de uma praia que se encontra em processo de erosão.

O MEPPE desperta a curiosidade do usuário e incentiva a aplicação, na prática, dos conceitos teó-ricos adquiridos, devido à facilidade de aplicação do modelo e à visualização gráfica dos resultados.

O sistema funciona da seguinte maneira:

1.O usuário carrega na tela do computador, um mapa ou uma fotografia aérea, em formato Raster (extensões bmp, jpg), de uma determinada praia (Figura 6);

2.Com a imagem na tela devem ser selecionadas as opções que indicam ao MEPPE a disposição da praia e o promontório em relação à tela (se a faixa de areia da praia está para cima ou para baixo, etc.);

3.O usuário deve selecionar na imagem, utilizando o mouse, três pontos, segundo o Modelo Parabólico: (1) o ponto de difração das ondas que chegam à praia; (2) o ponto final da praia e (3) a direção predominante das ondas;

4.Neste momento o usuário pode calcular e 
simular os resultados na tela.

O sistema apresenta então o valor do ângulo (beta), os valores de todos os ângulos teta, seus respectivos raios $\mathrm{R}_{n}$ e o comprimento do raio $\mathrm{R}_{0} \mathrm{O}$ mais importante é que ele desenha sobre a imagem da praia a linha teórica de costa, isto é, desenha onde deveria estar a linha de costa, em relação aos pontos indicados pelo usuário. Assim, define-se a condição em que a praia está, em equilíbrio estático ou equilíbrio dinâmico, dependendo da localização e formato da linha teórica de costa em relação à linha de costa existente. Em equilíbrio estático, a linha de costa teórica coincide com a linha de costa observada na imagem.

O usuário pode testar outras situações para a mesma praia, como alterar a posição do ponto de di-fração, simulando, talvez, a construção de um molhe ou quebra-mar. Neste caso, o MEPPE desenha nova-mente a Linha de Costa Teórica e apresenta todos os resultados para essa nova situação. Desta maneira, o usuário pode exercitar a aplicação do modelo, com-provando a teoria, o que lhe ajudará no aprendizado do tema.

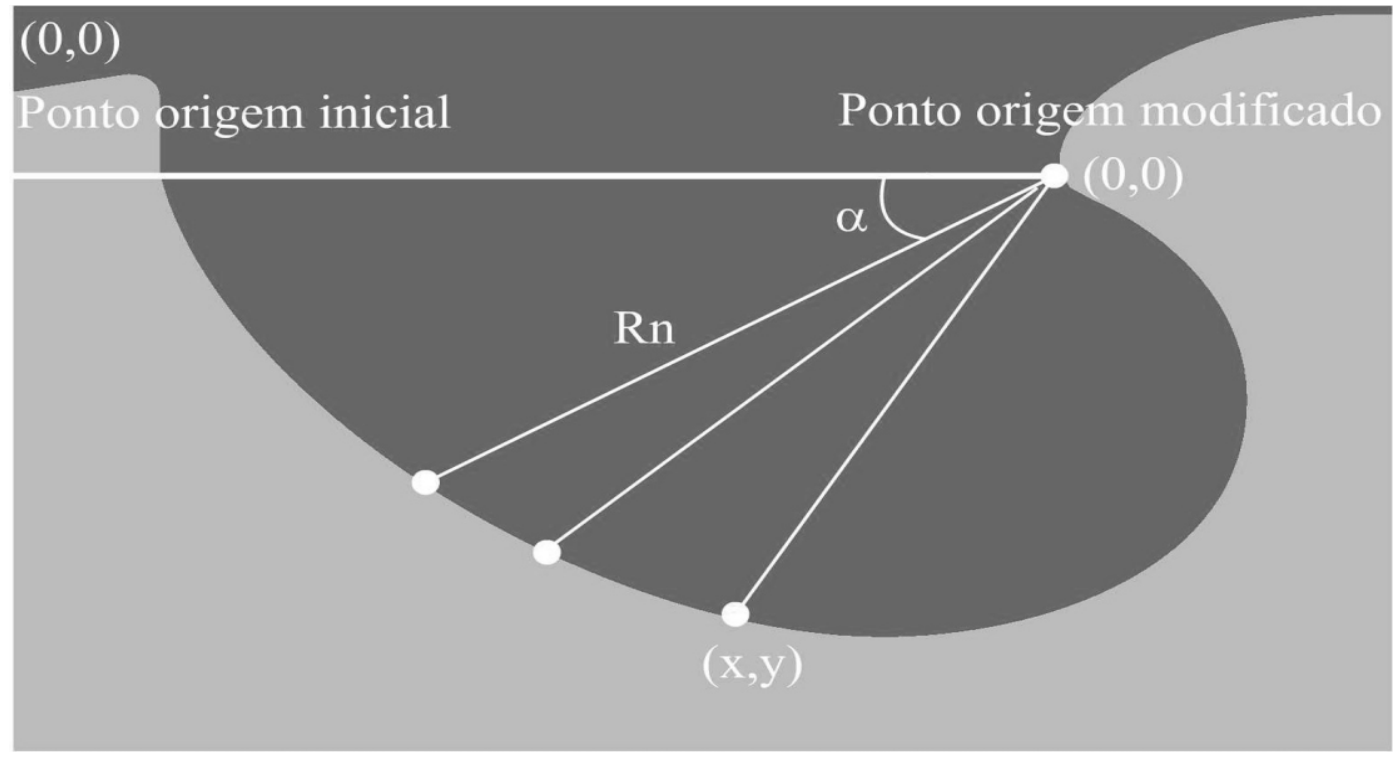

Figura 5. Localização da linha teórica de costa.

\section{Considerações Finais}

O MEPPE foi desenvolvido com o objetivo de se constituir numa ferramenta de auxílio aos educadores e educandos no ensino e aprendizagem do modelo parabólico de praias arenosas e sua aplicação prática. Ele não tem por finalidade a substituição da inteligência dos educadores e educandos, mas sim, apenas contribuir e propiciar o estímulo e o desenvolvimento de tal inteligência. Mesmo com o uso do MEPPE torna-se necessário que o usuário tenha conhecimento da teoria do modelo, para posteriormente utilizá-lo e comprovar essa teoria, sendo por isso importante o acompanhamento de um tutor.

Com o MEPPE simulando o resultado da aplicação do Modelo Parabólico fica mais simples o aprendizado e a análise das situações de Equilíbrio Estático ou Equilíbrio Dinâmico apresentadas pelas praias de enseada.

A possibilidade de testar diversas posições diferentes para os pontos importantes definidos pelo modelo, auxilia, não somente na compreensão da aplicabilidade do mesmo, como também na sua pró-pria definição. Além disso, o MEPPE pode também ser utilizado como ferramenta de testes em projetos de modificação do ponto de difração das ondas, como a construção de molhes ou quebra-ondas, pre-vendo possíveis danos ou benefícios.

O sistema encontra-se disponível gratuita-mente na Internet no endereço eletrônico

Http://www.cttmar.univali.br/ meppe

\section{Agradecimentos}

Os autores agradecem ao Prof. Dr. Rafael Carlos Vélez Benito, pelo auxílio nas questões matemáticas envolvidas na realização do trabalho, e aos Prof. Fernando Luiz Diehl, MSc. e Prof. Fabiane Barreto Vavassori, MSc. pelas correções e sugestões ao manuscrito inicial. 


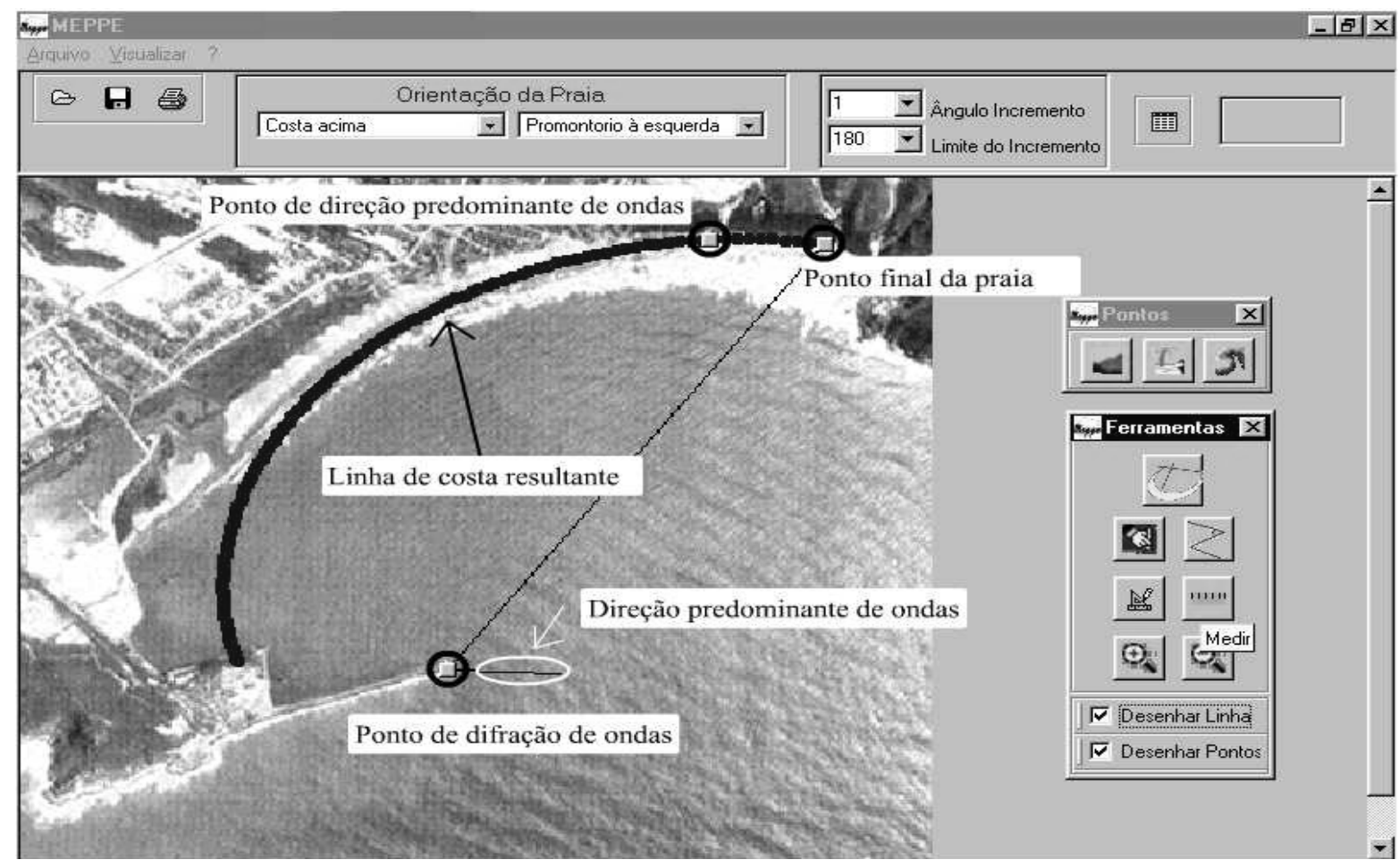

Figura 6. Ilustrando a utilização do MEPPE. Observa-se a seleção do ponto de difração e da direção predominante de ondas pelo usuário, e o desenho da linha de costa pelo programa. Utilizou-se uma foto aérea da Praia de Imbituba, localizada no litoral do Brasil, mais precisamente no Estado de Santa Catarina. Nesta simulação pode-se perceber que a praia encontra-se em Equilíbrio Dinâmico, pois sua linha de costa tende a ser modificada pelo fato do ponto de difração de ondas ter sido modificado pela construção de um quebra-mar.

\section{Bibliografia}

Anton, H. (2000). Cálculo, um novo horizonte. Trad. Cyro de Carvalho, Pataria e Márcia Tamanaha. 6. ${ }^{\mathrm{a}}$ ed. Bookman, Porto Alegre, $658 \mathrm{p}$.

Boulos, P. E Camargo, I. (1987). Geometria analítica: um tratamento vetorial. Makron Books, São Paulo: Mac. Graw-Hill, 385p.

Cantù, M. (2000). Dominando o Delphi 5 A Bíblia / Marco Cantù; Tradução João E.N. Tortello. São Paulo: MKRON Books, 861p.

Giraffa, L.M.M. (2000). Uma Arquitetura de Tutor utilizando Estados Mentais. Tese de Doutorado. Pós-Graduação em Ciência da Computação UFRGS, 98p.

Hoefel, F. G. (1998). Diagnóstico da Erosão Costei-ra na Praia de Piçarras, Santa Catarina. Dissertação de Mestrado. Universidade Federal do Rio de Janeiro, 86p.

Hsu, J.R.C., E Evans, C. (1989). Parabolic bay shapes and applications. Proceedings Institu-tion of Civil Engineers, Part 2. London: Thomas Telford, 87, 557-570.

Klein, A.H.F; Benedet Filho, L. and Hsu, J.R. C. Stability of Headland Bay Beaches in Santa Catarina: a Case Study. Journal of Coastal Research, SI (35). (em publicação).

Moreno, L.J., E Kraus, N.C. (1999). Equilibrium shape of headland-bay beaches for engineering design. Proceedings, Coastal Sediments '99. American Society of Civil Engineers, 1, p. 860-875.

Raabe, A.L.A.; Javimczic, A.M.; Giraffa, L.M. M. (1996). Eco-Lógico: Ambiente Interativo para Suporte ao Ensino de Educação Ambiental. Simpósio Brasileiro De Informática Na Educação (7.:1996 : Belo Horizonte). Anais p. 45-54. Belo Horizonte.

Short, A. D. E Masselink, G (1999). Embayed and structurally controlled beaches. In Handbook of Beach and Shoreface Morfodynamics, (ed. A.D. Short), pp 230249.

Silvester, R. (1970). Coastal Defense". Proceedings Institution of Civil Engineers., 45:677-682.

Silvester, R. E Hsu, J.R.C. (1993). Coastal Stabilization: innovative concepts. Englewood Cliffs, New Jersey . Prentice Hall, 578p.

Valente, J. A. (1989). Questão do Software: Parâmetros para o Desenvolvimento de Software Educativo. Núcleo de Informática Aplicada à Educação Memo No. 24 Universidade Estadual de Campinas: $\begin{array}{llllllll}\mathrm{U} & \mathrm{N} & \mathrm{I} & \mathrm{C} & \mathrm{A} & \mathrm{M} & \mathrm{P} & \text {. }\end{array}$ [http://www.nied.unicamp.br/]. (4 maio 2000 23:00). 